Economía, Sociedad y Territorio, vol. xx, núm. 64, 2020, 865-898.

DOI:https://doi.org/10.22136/est20201576

\title{
Modelo Analítico Jerárquico para la evaluación de factores turísticos en playas
}

\section{Analytical Hierarchy Model for the assessment of factors of beach tourism}

\author{
Cesar Augusto Hoil Rosas* \\ José Juan Carbajal Hernández* \\ Luis Pastor Sánchez Fernández* \\ Virginia Berenice Niebla Zatarain** \\ Alberto Daniel Dávila Lamas*
}

\begin{abstract}
Mexico is one of the most important countries on international tourism, being this activity of great importance due to the annual income it produces. However, technological resources employed in this field are few, thus stagnating the growth of tourism mainly in coastal areas. This work proposes the design of a computational model based on the Analytic Hierarchy Process to obtain a Beach Assessment Index that issues recommendations for tourism activities. The purpose of this index is to provide relevant information for tourists who are preparing to travel and stay on Mexican beaches.
\end{abstract}

Keywords: Analytic Hierarchy Process, tourism, beach, signal processing, assessment.

\section{Resumen}

México es uno de los mayores países en captar turismo internacional, siendo esta actividad de gran importancia debido a los ingresos económicos que produce. Sin embargo, pocos son los recursos tecnológicos empleados en este campo, estancando así el crecimiento del turismo principalmente de las zonas costeras. Este trabajo propone el diseńo de un modelo computacional basado en un Proceso Analítico Jerárquico, a fin de obtener un índice de evaluación de playas que emita recomendaciones para la realización de actividades turísticas. Este índice tiene como finalidad proporcionar información relevante para turistas que se disponen a viajar con el propósito de realizar estancias en playas mexicanas.

Palabras clave: Proceso Analítico Jerárquico, turismo, playa, procesamiento de señales, evaluación.

* Centro de Investigación en Computación, Instituto Politécnico Nacional, correos-e: cesarhoil_92@hotmail.com, carbajalito@hotmail.com, lsanchez@cic.ipn.mx, addavlam@gmail.com

** Instituto Tecnológico de Estudios Superiores de Los Cabos, correo-e: vbniebla@hotmail.com 


\section{Introducción}

México es uno de los principales líderes del turismo internacional receptivo, pues ocupa el sexto lugar a nivel mundial con más de 20 millones de turistas ingresados por año desde comienzos del siglo XXI (Benseny, 2007); genera, según datos de la Organización Mundial del Turismo, 8.2\% del PIB nacional en 2018 , del cual $45 \%$ fue producido por las zonas costeras (UNWTO, 2018). Esta posición se debe a la gran variedad de productos turísticos que ofrece el país, desde el clásico turismo de sol y playa hasta el turismo arqueológico (Propin y Sánchez, 2002). En México, el turismo se ha convertido en un factor determinante en ámbitos como la política, economía y sociedad (Rodríguez, 2015), tan es así que el Plan Nacional de Desarrollo 2013-2018 subraya la importancia de provechar el potencial turístico de México a fin de generar una mayor derrama económica en el país (DOF, 2013).

Con todo y sus implicaciones, el Estado mexicano califica al turismo como una actividad clave para el desarrollo y la generación de empleos. Debido a lo anterior, el gobierno busca promover y apoyar al turismo por medio de la expansión de la oferta turística y actualizar esta actividad a través de la implementación de los Destinos Turísticos Inteligentes (Sectur, 2018). Sin embargo, para lograr el desarrollo exitoso de los Destinos Turísticos Inteligentes (DTI), es necesario que México considere y mejore varios aspectos, siendo uno de los más importantes la creación de modelos matemáticos que sean capaces de gestionar, evaluar y clasificar factores turísticos con la finalidad de administrar de manera eficiente las playas de gran concurrencia y ofrecer así una experiencia más placentera a los turistas (Pérez, 2011). Actualmente, la Secretaría de Medio Ambiente y Recursos Naturales (Semarnat, 2019) realiza programas de análisis de sanidad en playas en las cuales estudia la cantidad de microorganismos; se enfoca en las coleiformes fecales y enterococos y orienta a la población a seleccionar la playa que se encuentre más limpia entre todas las analizadas.

En la literatura se pueden encontrar proyectos que se enfocan en clasificar zonas turísticas de acuerdo con determinadas variables, como son la urbanización y el riesgo, entre otras. En el trabajo realizado por Ranieri y El-Robrini (2016) se analizan las condiciones oceanográficas en las playas de Salinópolis, Brasil, y actividades relacionadas con el ocio y la ocupación humana; el objetivo es categorizar en términos de urbanización. Petrosillo et al. (2010) realizaron una evaluación subjetiva de los riesgos ambientales asociados con un puerto turístico al sur de Italia y compararon los resultados para proporcionar estimaciones de seguridad ambiental. En Brasil, Cristiano et al. (2016) evaluaron el paisaje costero del municipio de Torres, con base en 26 parámetros naturales y humanos 
para clasificar las playas en 5 clases, siendo la clase 1 la de mayor atractivo paisajístico y la clase 5 la menos atractiva. En el estudio propuesto por Ergin et al. (2004), se utilizaron 26 parámetros para evaluar los paisajes de las costas en Turquía y Reino Unido, a través de encuestas a turistas y expertos en percepción del paisaje. Después, Ergin et al. (2010) evaluaron 34 zonas costeras en la región occidental del Mar Negro. A su vez, Phillips y House (2009) analizaron las prioridades del turismo en la playa por medio de la asignación de pesos obtenidos por encuestas. Estos trabajos sirven de apoyo en nuestra investigación, debido a que todos contemplan el uso de la percepción para obtener evaluaciones de cada criterio para establecer si una playa es mejor que otra. Todos estos análisis proporcionan pesos y puntuaciones para conocer cuál es el mejor aspecto rankeado. Sin embargo, hay un factor en contra y es el hecho de que evaluar tantos aspectos en una playa de manera continua se hace prácticamente imposible, además de que hay que buscar por separado aquellos que mejor se evaluaron. Otro problema es que este tipo de evaluaciones son muy espaciadas debido a su complejidad y no reflejan una situación actualizada del sitio. Por una parte, este trabajo propone realizar un índice que considere varios parámetros para evaluar un aspecto de interés y, por otro lado, recomienda estudiar parámetros que pueden automatizarse, ya que éstos permitirán consultar el estado de la playa en cualquier instante a diferencia de los propuestos en la literatura, en los cuales se tiene que esperar la evaluación completa de un gran conjunto de criterios antes de poder tener una evaluación.

En lo que respecta a México, Troyo et al. (2018) examinaron el servicio turístico en el avistamiento de la ballena gris en Baja California Sur y utilizaron un modelo basado en estadística para realizar dicha evaluación. Aunque en este trabajo se propone un índice integral, no está diseñado para la evaluación de playas como tal, por lo que su implementación nos brinda una opción parcial.

Existen diversas investigaciones que han ayudado a obtener nuevas formas de procesar el Proceso de Jerarquía Analítica (AHP, por sus siglas en inglés: Analytical Hierarchy Process). Entre ellas se pueden mencionar los trabajos propuestos por Adamopoulos y Pappis (1996), Chang (1996), Dağdeviren y Yüksel (2008) y Wong y Li (2008), quienes utilizan modelos basados en el análisis multicriterio y una adaptación difusa conocida como FAHP. Aunque son propuestas interesantes, presentan metodologías más complejas sobre el uso del AHP para problemas de decisión. En el caso de la obtención de pesos de importancia, el modelo eje propuesto por Thomas Saaty (2008) muestra resultados muy similares a los modelos reportados en la literatura. No obstante, la simplicidad del modelo AHP 
permite entender con mayor facilidad el proceso de cálculo del índice, lo cual es parte de la motivación de este trabajo.

Con base en lo anterior, este artículo tiene como objetivo establecer un marco de evaluación sobre las condiciones apropiadas para el turismo en los litorales de Baja California Sur, México. Como contribución, se diseña un índice de evaluación de costas que utiliza un modelo de prioridades basado en el AHP y el procesamiento de parámetros climáticos y oceanológicos que puedan monitorearse de forma automatizada, tales como temperatura, oleaje, radiación solar, humedad y velocidad del viento. Este índice tiene como principal ventaja el adaptarse a las diferentes actividades de mayor demanda por turistas que visitan una misma zona, ya que permitirá proporcionar información sobre qué sitio presenta las mejores condiciones de acuerdo con dicha actividad.

\section{1. Área de Estudio}

El presente trabajo analizó las playas de Baja California Sur, en donde se pueden encontrar diversas costas con características completamente diferentes entre ellas. Se planteó el estudio de la bahía de La Paz y la playa Costa Azul en San José del Cabo, debido a que son dos de las playas más visitadas en Baja California Sur; la primera presenta un bajo oleaje y segunda tiene características de ser mar abierto.

\subsection{La Paz}

La bahía de La Paz se encuentra situada en el suroeste del golfo de California entre los paralelos $24^{\circ} 45^{\prime}$ y $24^{\circ} 15^{\prime}$ norte y los meridianos $110^{\circ}$ $15^{\prime}$ y $110^{\circ} 45^{\prime}$ oeste. Adicionalmente, la bahía constituye el cuerpo de agua más extenso en la costa oriental de la península de Baja California con una superficie aproximada de $1780 \mathrm{~km}^{2}$ y un perímetro costero de $200 \mathrm{~km}$ de longitud (Abitia-Cárdenas et al., 1994). Entre sus características, posee una temperatura mínima superficial en la bahía de $20{ }^{\circ} \mathrm{C}$ en invierno-primavera y la máxima de $31^{\circ} \mathrm{C}$ en verano (Espinoza y Rodríguez, 1987). La Paz es un punto vacacional que cuenta con una amplia variedad de diversión y entretenimiento debido a sus espléndidas playas, islas y su inmensa variedad de especies animales y vegetales, lo que ha causado que sea la región perfecta para la práctica de turismo ecológico. En esta zona se encuentra ubicado el Centro Interdisciplinario de Ciencias Marinas (Cicimar), que pertenece al Instituto Politécnico Nacional, dedicado al estudio de este lugar, que fue utilizado para albergar equipo tecnológico de medición de parámetros. 


\subsection{San José del Cabo}

San José del Cabo es una ciudad turística que se ubica en el extremo sur de la Península de Baja California entre los paralelos $23^{\circ} 01^{\prime}$ y $23^{\circ} 04^{\prime}$ norte y los meridianos $109^{\circ} 50^{\prime}$ y $109^{\circ} 58^{\prime}$ oeste, a $180 \mathrm{~km}$ de la ciudad de La Paz, capital del estado (Martínez y Díaz, 2011). Al este de la ciudad se encuentra el Estero de San José, una reserva de la naturaleza con especies de aves, como colibríes, ibis y garzas (figura 1).

\section{Figura 1}

\section{Localización de San José del Cabo y La Paz, Baja California Sur}

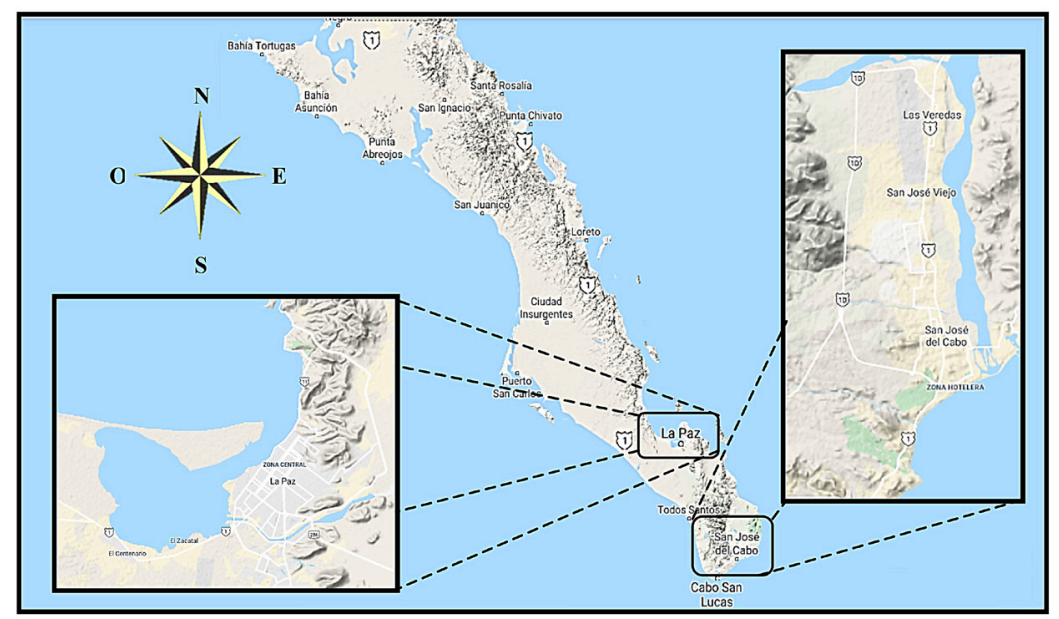

Fuente: elaboración propia con base en Google Maps (2019).

Entre las principales actividades que se realizan en la región están las caminatas artísticas, surf y buceo en alguna de sus maravillosas playas y el avistamiento de aves en el Estero de San José. En esta localidad, el Instituto Tecnológico de Estudios Superiores de los Cabos y la Zona Federal Marítimo Terrestre (Zofemat, 2019) proporcionaron apoyo en sus instalaciones, así como la ayuda de expertos en turismo que ayudaron a establecer las prioridades entre parámetros y factores en esta zona. La playa de Costa Azul fue elegida para realizar el estudio debido a que es considerada una de las mejores playas para la práctica de surf (Pérez et al., 2016). Zofemat cuenta con alrededor de seis módulos de vigilancia distribuidos en las diversas playas de San José; su principal trabajo es salvaguardar el área correspondiente a los 20 metros de distancia contigua al litoral marítimo. 


\section{Materiales y métodos}

Hoy en día, existen diversos criterios para establecer las actividades que se pueden realizar en una zona determinada, los cuales se basan en conocer las condiciones ideales de un conjunto de parámetros mediante una selección de importancia entre un criterio y otro. El proceso de Jerarquía Analítica (AHP) permite establecer diferencias entre parámetros y otros factores de interés que definen si una playa es atractiva o no. En este trabajo se considera monitorear parámetros físicos, involucrados en diferentes factores asociados a una playa, con la intención de tener un modelo de supervisión constante y preciso. El desarrollo de este proyecto se conforma principalmente de tres etapas. La primera es la definición del objetivo que para este caso es el seleccionar una playa adecuada. Posterior a esto, se puntualizan los criterios de evaluación, estableciendo las prioridades con base en la información proporcionada por el ITES, Los Cabos y la Zofemat (2019), cuyo orden de importancia dependerá de los siguientes factores: seguridad, recreación familiar, actividades acuáticas y desahogo. Éstos permitirán identificar qué tipo de actividad es más conveniente según el lugar. Asimismo, se establecen las prioridades de los parámetros de acuerdo con cada actividad y factor. Finalmente, se proponen las diversas alternativas para evaluar cada playa.

\subsection{Análisis de factores}

Los factores de análisis son de gran relevancia para solucionar un problema y éstos a su vez constan de varios parámetros que definen su importancia. En este proyecto se consideran cuatro factores principales que los turistas toman en cuenta para visitar una playa, basados en las sugerencias emitidas por investigadores del Instituto Tecnológico de Estudios Superiores de Los Cabos, que se enlistan a continuación.

\section{F1: Recreación familiar}

Los principales visitantes de playas son familias que tienen como prioridad divertirse en conjunto. Debido a lo anterior, este factor se enfoca en los turistas que buscan en una playa aquellas características necesarias para llevar a cabo las diversas actividades que fomentan la convivencia y recreación entre jóvenes, niños y adultos.

\section{F2: Desahogo}

En la actualidad, el estrés es un problema que afecta a la mayoría de las personas, por lo cual en diversas ocasiones los turistas deciden visitar una playa con el único objetivo de relajarse en un ambiente de serenidad. Este 
factor se enfoca en esta clase de turistas, los cuales tienen como prioridad la relajación.

\section{F3: Seguridad}

Uno de los principales factores que los turistas toman en cuenta para elegir un lugar para vacacionar es la seguridad, el cual es fundamental para garantizar la protección a los visitantes que desconocen la zona.

\section{F4: Actividades acuáticas}

Las actividades acuáticas son aquellas disciplinas en las que el deportista realiza una actividad física en el agua. Con base en lo anterior, este factor se enfoca principalmente en los turistas cuya prioridad principal es encontrar las condiciones ideales de una playa para llevar a cabo actividades como el surf, esquí acuático, kitesurf, entre otros más.

\subsection{Análisis de parámetros}

Existen diferentes parámetros de gran interés para el turista, que pueden categorizarse como físicos, biológicos o urbanos, los cuales definen las características del lugar al que se desea visitar (tabla 1). En el análisis de preferencias y recomendaciones para la visita turística, los parámetros siempre están relacionados con los factores, toda vez que ellos definirán el nivel de importancia de cada uno de ellos. En este trabajo, los parámetros físicos son el conjunto de estudio, ya que pueden automatizarse para obtener información sobre ellos continuamente, y debido a que éstos varían constantemente dependiendo de las condiciones meteorológicas del lugar, se hace necesario su estudio para identificar la condición del sitio recreativo.

\section{Tabla 1}

\section{Tipos de parámetros involucrados en el estudio de preferencias}

\begin{tabular}{ccc}
\hline Físicos & Biológicos & Urbanos \\
\hline Temperatura & Color del agua & Ruido \\
Oleaje & Tipo de arena & Salvavidas \\
Radiación solar & Paisaje & Basura \\
Humedad & Tipo de vegetación & Acceso \\
Velocidad del viento & Microorganismos & Infraestructura \\
\hline
\end{tabular}

Fuente: elaboración propia con base en Pérez et al. (2008). 
De igual forma, se establecen los niveles máximos o mínimos de cada parámetro que se deben presentar para obtener un nivel de seguridad y aceptación para el turista. En la tabla 2 se presenta la clasificación de los parámetros con base en su rango óptimo, así como las tolerancias para su medición. Estos valores fueron obtenidos de otros trabajos de investigación especializados en turismo.

Tabla 2

Valores óptimos y límites difusos de cada parámetro

\begin{tabular}{lcccccc}
\hline \multirow{1}{*}{ Parámetro } & Rango & Unidades & \multicolumn{4}{c}{ Limites tolerables } \\
\cline { 4 - 7 } & Óptimo & & $a$ & $b$ & $c$ & $d$ \\
\hline Temperatura $(t)$ & $25-30$ & ${ }^{\circ} \mathrm{C}$ & 22 & 25 & 30 & 33 \\
Oleaje $(o)$ & $0.1-0.5$ & $m$ & 0 & 0.5 & 1 & 0 \\
Radiación solar $(r)$ & $6.1-10.1$ & $\mathrm{~kW} / \mathrm{m}^{2}$ & 6.1 & 8.1 & 10.1 & 0 \\
Humedad $(h)$ & $40-70$ & $\%$ & 40 & 55 & 70 & 0 \\
Velocidad del viento $(v)$ & $4-12$ & $\mathrm{Km} / \mathrm{hr}$ & 1 & 4 & 12 & 16 \\
\hline
\end{tabular}

Fuente: elaboración propia con base en Kim et al. (2004), Wright (2008) y Cengel y Boles (2006).

\subsection{Evaluación difusa de parámetros}

La lógica difusa ha sido desarrollada como una forma de evaluar variables con base en el enfoque del vaso medio lleno o medio vacío. Es decir, cuando algún parámetro tiende a evaluarse entre un estado falso o verdadero, el resultado será un valor dentro de un rango 0 a 1 , lo cual significa que la variable puede tener un nivel de un estado u otro. Este enfoque es de gran importancia, ya que todos los parámetros presentan niveles diferentes y la lógica difusa permite transformar estos valores en rangos uniformes. Es importante subrayar que cada parámetro a analizar en este trabajo tiene un diferente rango de valores, por lo que es necesario normalizarlos para procesarlos en la misma escala de medición. Para llevar a cabo esto, se propone la evaluación mediante funciones difusas, que permitirán normalizar los niveles de cada parámetro en rangos de [0-1]. Las siguientes funciones difusas son definidas para cada parámetro, con base en los límites tolerables $(a, b, c, d)$ establecidos en la tabla 2 . 


$$
\begin{aligned}
& \mu(\lambda)=\max \left\{\min \left(\frac{x-22}{25-22}, 1, \frac{33-x}{33-30}\right), 0\right\}(1) \quad \mu(0)=\max \left\{\min \left(1, \frac{1-x}{1-0.5}\right), 0\right\} \\
& \mu(r)=\max \left\{\min \left(\frac{x-6.1}{8.1-6.1}, 1, \frac{10.1-x}{10.1-8.1}\right), 0\right\}(3) \quad \mu(h)=\max \left\{\min \left(\frac{x-40}{55-40}, 1, \frac{70-x}{70-40}\right), 0\right\} \\
& \mu(\nu)=\max \left\{\min \left(\frac{x-1}{4-1}, 1, \frac{16-x}{16-12}\right), 0\right\}(5)
\end{aligned}
$$

\subsection{Proceso Analítico Jerárquico (AHP)}

El modelo AHP desarrollado en este proyecto se conforma principalmente de cuatro etapas. La primera consiste en la definición del objetivo; en este caso es seleccionar una playa adecuada. Posterior a esto, se puntualizan los factores principales, definidos por el tipo de actividad a realizar. Se identifican aquellos parámetros involucrados en dichas actividades que servirán de apoyo en el análisis del sitio de estudio. Después, se establecen criterios de importancia mediante la comparación y asignación de niveles de importancia entre parámetros y factores con base en la información proporcionada por el conjunto de expertos. Finalmente, se realiza el cálculo matemático para obtener los pesos correspondientes a cada parámetro y así obtener el índice final.

La asignación de pesos dirigida a algún tipo de parámetro no es una tarea clara y suelen asignarse en muchos de los casos de forma discrecional. El Proceso Analítico Jerárquico es una metodología propuesta por Thomas Saaty (2008), que permite crear pesos con base en una asignación subjetiva de importancia entre objetos mediante una tabla de niveles de importancia (tabla 3). En nuestro caso, permitirá fijar relaciones entre los parámetros de estudio de acuerdo con su impacto en cada factor turístico determinado. El proceso matemático empleado en este trabajo se muestra a continuación.

\section{Tabla 3}

\section{Escala propuesta por Saaty y Wind}

\begin{tabular}{ccccccccccc}
\hline $\begin{array}{c}\text { Nivel de } \\
\text { Importancia }\end{array}$ & Igual & + Moderado & + Fuerte & + Muy Fuerte & + Extremo \\
\hline Escala & 1 & 2 & 3 & 4 & 5 & 6 & 7 & 8 & 9 \\
\hline
\end{tabular}

Fuente: elaboración propia con base en Saaty y Wind (1980). 
El primer paso de este método consiste en generar comparaciones entre elementos en donde se asigne un criterio de importancia entre ellos como sigue:

$R$ : el elemento $A$ es moderadamente más importante que el elemento $B$.

Después de seleccionar el criterio, se convierte a su valor numérico y se crea una matriz pareada $(A=a i j)$, cuyos niveles de importancia entre parámetros son comparados (wawb). Esta matriz es cuadrada, recíproca y positiva y se construye como se muestra a continuación:

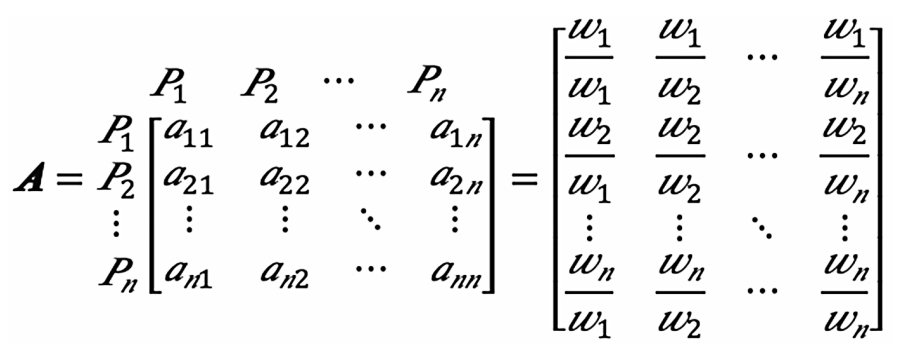

Una vez elaborada la matriz, se procede a calcular lo que se denomina como eigenvector (Perron, 1907), el cual ayudará a determinar los pesos. Éstos serán usados para crear el Índice de Evaluación de Costas (IEC). Con base en lo anterior, se requiere calcular la matriz cuadrada B de la siguiente manera:

$$
\mathbf{B}=\left[\begin{array}{cccc}
a_{11} & a_{12} & \cdots & a_{1 n} \\
a_{21} & a_{22} & \cdots & a_{2 n} \\
\vdots & \vdots & \ddots & \vdots \\
a_{n 1} & a_{n 2} & \cdots & a_{n n}
\end{array}\right] \times\left[\begin{array}{cccc}
a_{11} & a_{12} & \cdots & a_{1 n} \\
a_{21} & a_{22} & \cdots & a_{2 n} \\
\vdots & \vdots & \ddots & \vdots \\
a_{n 1} & a_{n 2} & \cdots & a_{n n}
\end{array}\right] \text { (7) }
$$

Después, la sumatoria de las filas es determinada de acuerdo con:

$$
C_{i}=\sum_{j=1}^{n} \mathrm{~B}_{i j} \forall i=1,2, \ldots, n
$$

Finalmente, la importancia de los pesos es obtenida normalizando el vector $C_{i}$, como se muestra en la siguiente fórmula:

$$
W_{i}=\frac{C_{i}}{\sum_{j=1}^{n} C_{j}} \forall i=1,2, \ldots, n
$$


Una consideración importante que ofrece el método AHP es medir el grado de consistencia de la matriz de comparaciones pareadas, esto es, cuando las asignaciones de importancia entre parámetros son coherentes. Si el grado de consistencia es aceptable, puede continuarse con el proceso de cálculo y si es inaceptable, se debe rehacer la matriz. Este grado puede calculase como:

$$
C R=\frac{\lambda_{\max }-n}{(n-1) R I}
$$

Donde $\lambda_{\text {max }}$ es el máximo eigenvalor de la matriz pareada, $n$ es el tamaño de la matriz, $R I$ es el índice de consistencia aleatoria y $C R$ es el radio de consistencia. El valor de $R I$ depende del número de elementos que se compara, tal y como se muestra en la tabla 4.

Tabla 4

Índice de consistencia aleatoria

\begin{tabular}{ccccccccccc}
\hline $\begin{array}{c}\text { Tamaño } \\
\text { de la matriz }\end{array}$ & 1 & 2 & 3 & 4 & 5 & 6 & 7 & 8 & 9 & 10 \\
\hline RI & 0 & 0 & 0.52 & 0.89 & 1.11 & 1.25 & 1.35 & 1.4 & 1.45 & 1.49 \\
\hline
\end{tabular}

Fuente: elaboración propia con base en Toskano (2005).

El resultado obtenido por $C R$ nos indica que si éste excede de 0.10 , se tiene una matriz inconsistente. Por el contrario, si es menor o igual a 0.10 , la matriz tiene un grado de consistencia aceptable.

\subsection{Indice de Evaluación de Costas}

El Índice de Evaluación de Costas (IAHP) es un valor difuso en un rango de [0-1], el cual se ve afectado directamente por el peso de cada parámetro, generando un puntaje que indica qué tan buena o mala es la evaluación de la playa. La siguiente fórmula describe la forma de calcular el índice:

$$
I A H P=\sum_{i=1}^{n} \mu_{i}(k) W_{i}^{k}
$$

Donde $n$ es la cantidad de parámetros, $\mu_{i}(k)$ es el valor difuso obtenido de las ecuaciones definidala en la sección $3.1 ; k$ refiere al parámetro y $W$ 
es la importancia del peso calculado por el método de AHP. Al reemplazar cada parámetro en la ecuación 11, se obtiene la siguiente expresión:

$$
I A H P=\mu(t) W^{t}+\mu(o) W^{o}+\mu(h) W^{h}+\mu(v) W^{v}
$$

Donde $t$ se refiere a la temperatura, $o$ al oleaje, $r$ a la radiación solar, $h$ a la humedad y $v$ a la velocidad del viento. Finalmente, un resultado cercano a 1 será más recomendable para el lugar y por el contrario, otro más próximo a 0 será menos recomendable.

\section{Resultados y discusiones}

\subsection{Evaluación del sitio de estudio}

El objetivo principal de este trabajo es evaluar diferentes playas, de acuerdo con alguna actividad de interés. Debido a que todas las playas tienen elementos diferentes que las hacen especiales en el país (en específico las ubicadas en Baja California Sur), se hace necesario definir un modelo que permita integrar esas diferencias. El Proceso de Jerarquía Analítica permite crear relaciones entre características del lugar acorde con un conjunto de preferencias identificadas por expertos en el área. Podemos citar como ejemplo las bahías de La Paz, reconocidas especialmente por su tranquilidad. Un indicador que priorice esta característica seguramente aportará un buen análisis en este sitio y similares y, por otra parte, descalificará playas de mar abierto, como es el caso de San José del Cabo, cuyo oleaje suele resultar peligroso para las actividades de natación.

Para la asignación de niveles de importancia entre factores y parámetros, un instrumento de evaluación fue elaborado para obtener la opinión de turistas en los sitios de estudio (anexo A). Dicho instrumento consistió en una encuesta, la cual fue elaborada con apoyo del Instituto Tecnológico de Estudios Superiores de Los Cabos y avalada por el personal de la Zona Federal Marítimo Terrestre (Zofemat, 2019). Ambas instituciones acordaron estudiar dos preferencias turísticas de gran interés en la zona: turismo en un ambiente familiar (a) y actividades para surf (b).

Zofemat encuestó a 50 personas en la localidad del Costa Azul (perfil de surf), mientras que estudiantes del ITES a otros 50 turistas en la Bahía de La Paz (Perfil familiar). Los promedios correspondientes obtenidos por factor, así como por comparación entre parámetros conforme con el factor preponderante, fueron utilizados para construir las matrices pareadas. En este caso los resultados fueron redondeados para obtener los niveles de importancia definidos en la tabla 3. Un detalle a observar es 
que tanto las instituciones como las encuestas coincidieron en que la seguridad es primordial en cualquier aspecto para el turista. Sin embargo, se entiende que los surfistas tienen experiencia en nado a mar abierto, por lo que este factor se relaja un poco, derivando en una mayor relevancia una ubicación que permita la actividad de surf dentro de un radio de seguridad. Asimismo, se compararon los resultados obtenidos con el trabajo propuesto por Phillips y House (2009), en donde se realiza un estudio con 50 parámetros involucrados en el análisis de playas turísticas. $\mathrm{Al}$ analizar un promedio de los pesos asignados a parámetros involucrados en los factores de estudio, se cumple con un orden de importancia similar a los considerados en este trabajo. Las relaciones de comparación $(R)$ fueron expresadas en un lenguaje coloquial para posteriormente ser traducidas al lenguaje matemático respectivo del AHP. En el caso de una comparación para ambientes familiares, ejemplificamos las siguientes comparaciones:

R1: La seguridad es moderadamente más importante que la recreación familiar.

R2: La recreación familiar es fuertemente más importante que el desahogo familiar.

Para el caso de actividades de surf, se plantean las mismas comparaciones con los siguientes criterios:

R3: La seguridad es extremadamente más importante que la recreación familiar.

R4: El deshago familiar es fuertemente más importante que la recreación familiar.

Para el caso de las reglas 1 y 2 , tenemos que la escala de importancias nos marca un valor de 3 en el nivel moderado y un valor de 4 en el nivel fuertemente importante. Sin embargo, al realizar la misma comparación con enfoque en las actividades de surf, se obtiene en la regla 3 un valor de 8 , lo cual nos indica que es extremadamente más importante. Asimismo, la regla 4, que tiene un valor correspondiente a 5 , nos indica que es fuertemente más importante. Estos valores son ingresados en la matriz de comparaciones que se muestra en la tabla 5. El resto del conjunto de comparaciones puede elaborarse siguiendo el mismo proceso. Las tablas 5 y 6 muestran las relaciones definidas para los dos tipos de ambientes, cuyos valores almacenados corresponden a los niveles de importancia asignados entre factores. 
Tabla 5

Comparación de los factores para el ambiente familiar, con un $\mathrm{CR}=\mathbf{0 . 0 1 9 8}$

\begin{tabular}{lcccccc}
\hline \multicolumn{1}{c}{ Factores } & $F 1$ & $F 2$ & $F 3$ & $F 4$ & Normalizado & Eigenvector \\
\hline F1: Recreación familiar & 1 & 5 & $1 / 3$ & 4 & 0.2990 & 0.2669 \\
F2: Desahogo & $1 / 5$ & 1 & $1 / 9$ & $1 / 2$ & 0.0524 & 0.0533 \\
F3: Seguridad & 3 & 9 & 1 & 6 & 0.5497 & 0.5911 \\
F4: Actividades acuáticas & $1 / 4$ & 2 & $1 / 6$ & 1 & 0.0989 & 0.0887 \\
\hline & & & Suma: & 1 & 1 \\
\hline
\end{tabular}

Fuente: elaboración propia con base en las prioridades para la recreación familiar.

Tabla 6

Comparación de los factores para las actividades de surf, con un $\mathrm{CR}=\mathbf{0 . 0 5 1}$

\begin{tabular}{lcccccc}
\hline \multicolumn{1}{c}{ Factores } & $F 1$ & $F 2$ & $F 3$ & $F 4$ & Normalizado & Eigenvector \\
\hline F1: Recreación familiar & 1 & $1 / 5$ & $1 / 8$ & $1 / 9$ & 0.0341 & 0.0364 \\
F2: Desahogo & 5 & 1 & $1 / 5$ & $1 / 7$ & 0.1506 & 0.0975 \\
F3: Seguridad & 8 & 5 & 1 & $1 / 3$ & 0.3404 & 0.2935 \\
F4: Actividades acuáticas & 9 & 7 & 3 & 1 & 0.4749 & 0.5726 \\
\hline & & & & Suma: & 1 & 1 \\
\hline
\end{tabular}

Fuente: elaboración propia con base en las prioridades para las actividades acuáticas.

En una segunda etapa, se generan comparaciones con base en el parámetro contra el factor de importancia (10 comparaciones en cada caso). Las tablas 7, 8, 9 y 10 tienen las matrices pareadas resultantes de las comparaciones realizadas para cada parámetro conforme con el ambiente familiar y actividades de surf. Asimismo, en cada tabla se calculan los pesos mediante el método de normalización y eigenvector. El cálculo de los radios de consistencia de las matrices nos indican el nivel de consistencia de nuestras relaciones definidas en cada caso.

Para obtener los pesos finales de cada parámetro, es necesario combinar todos los resultados obtenidos mediante la multiplicación de los pesos obtenidos para cada factor, por los pesos individuales de cada parámetro y determinados por cada factor. Las tablas 11 y 12 muestran los valores finales obtenidos por el método de AHP. 


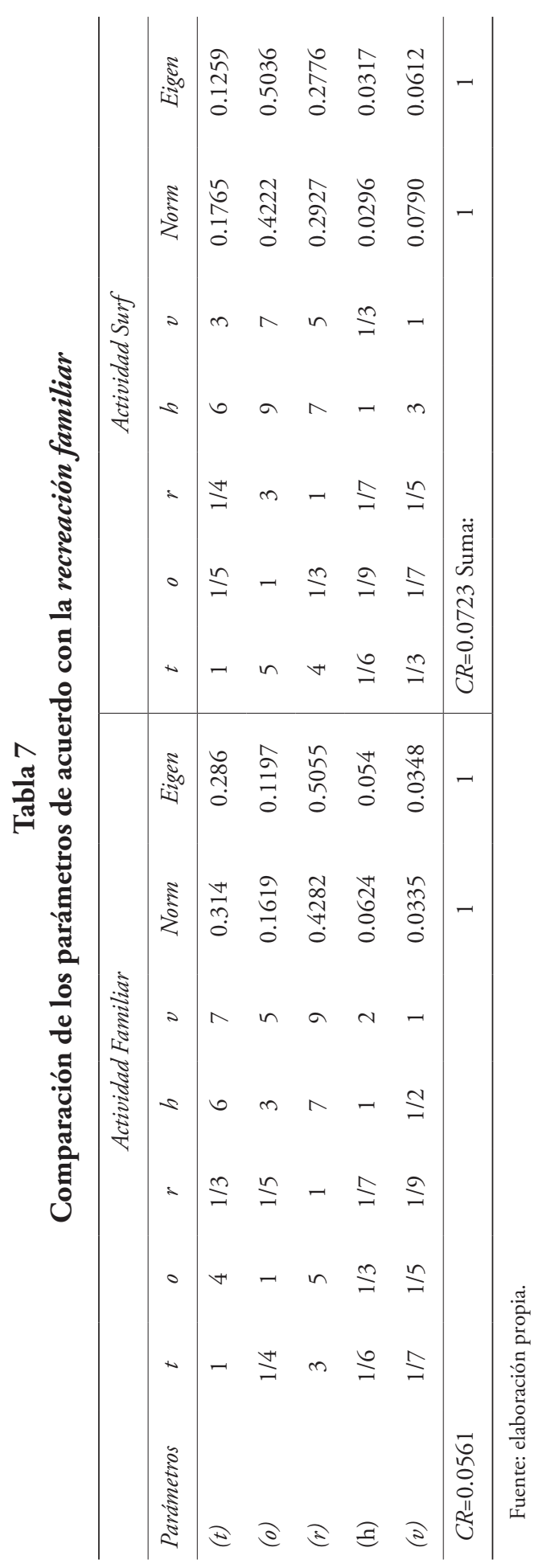




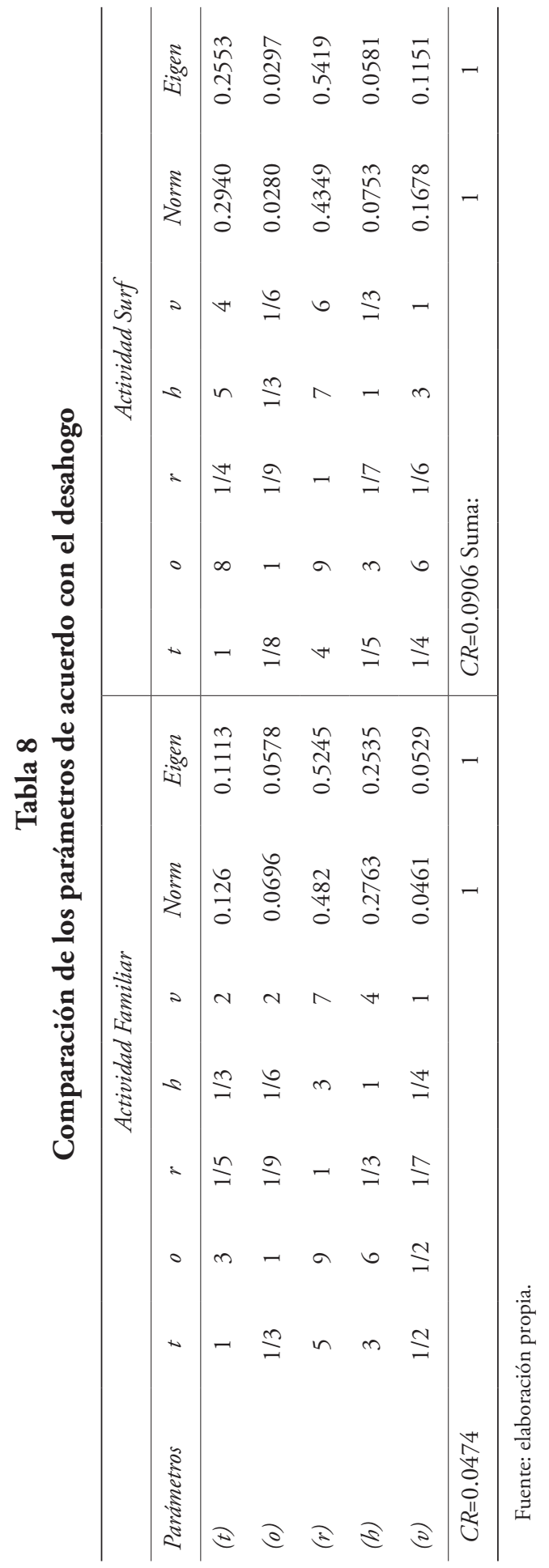




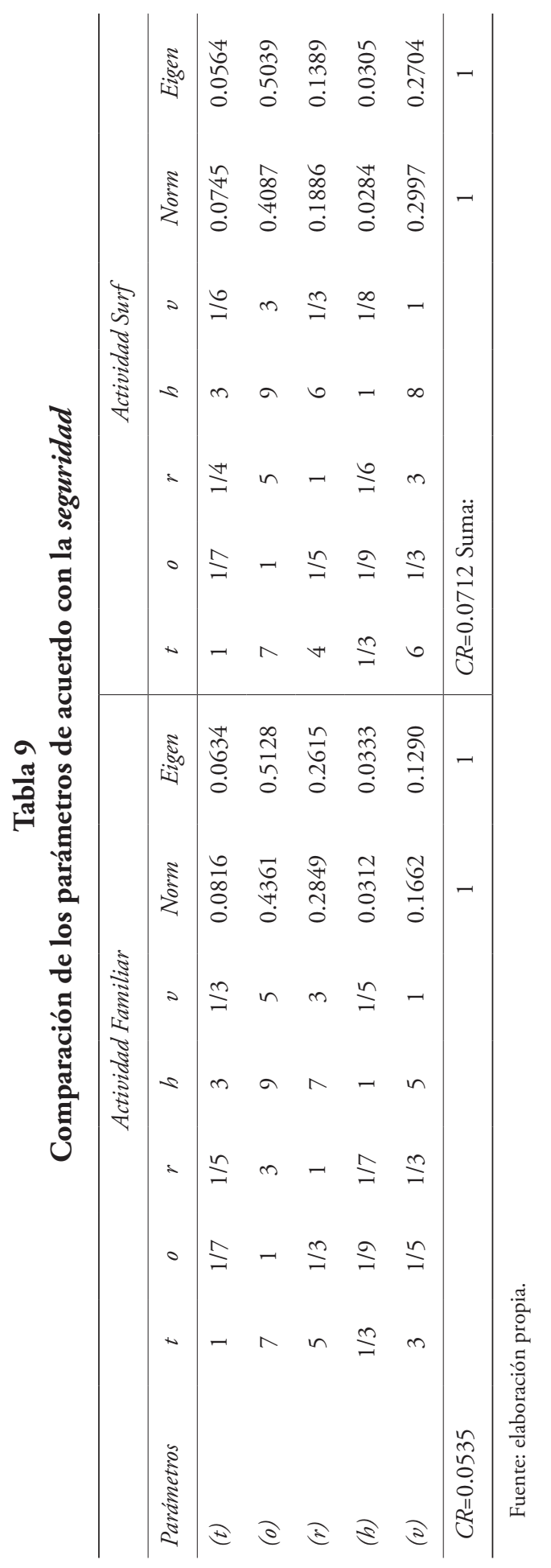




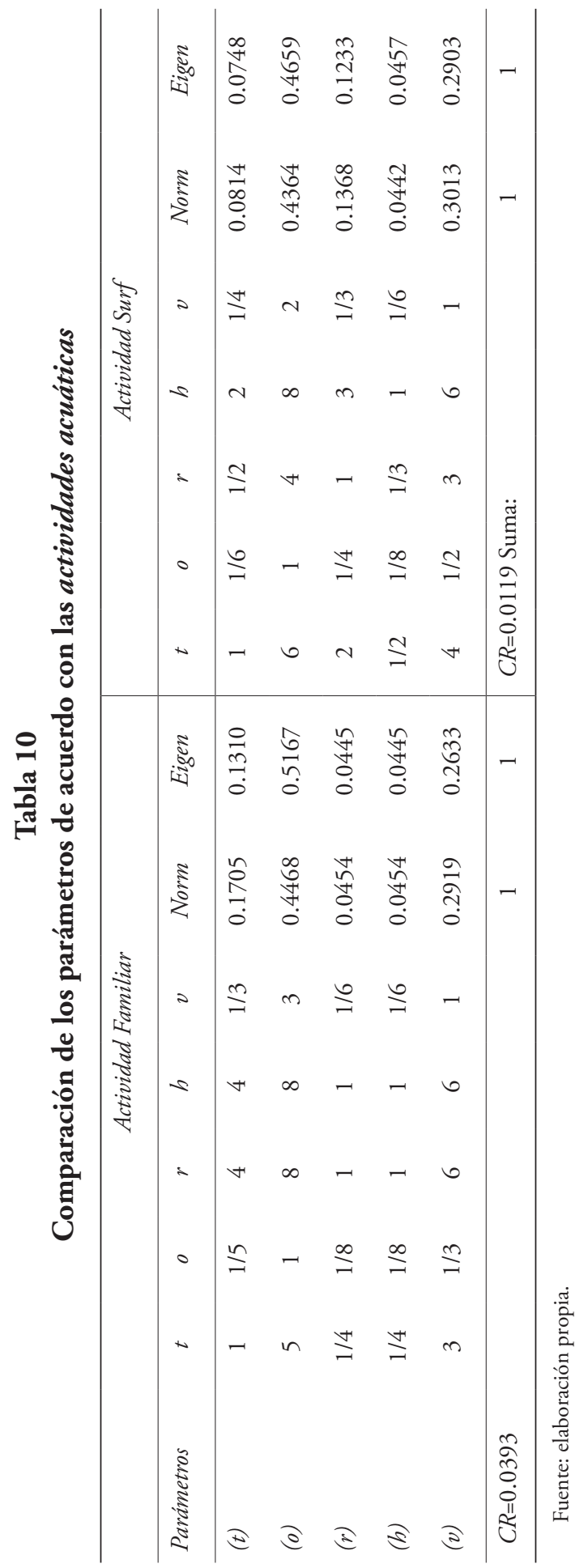




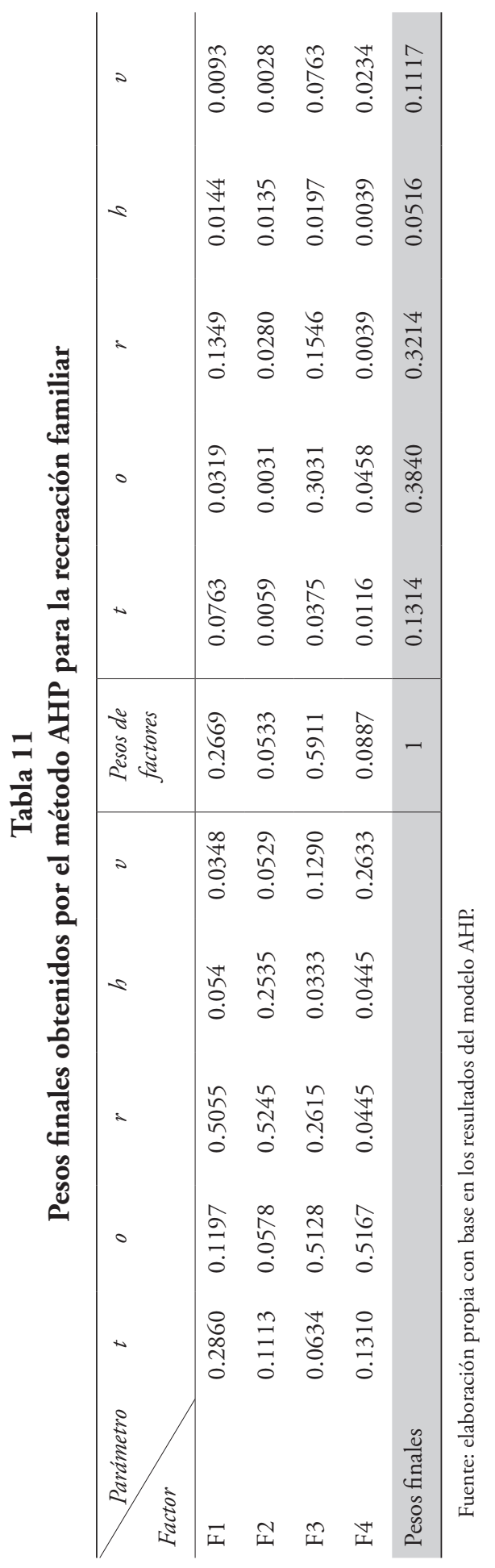




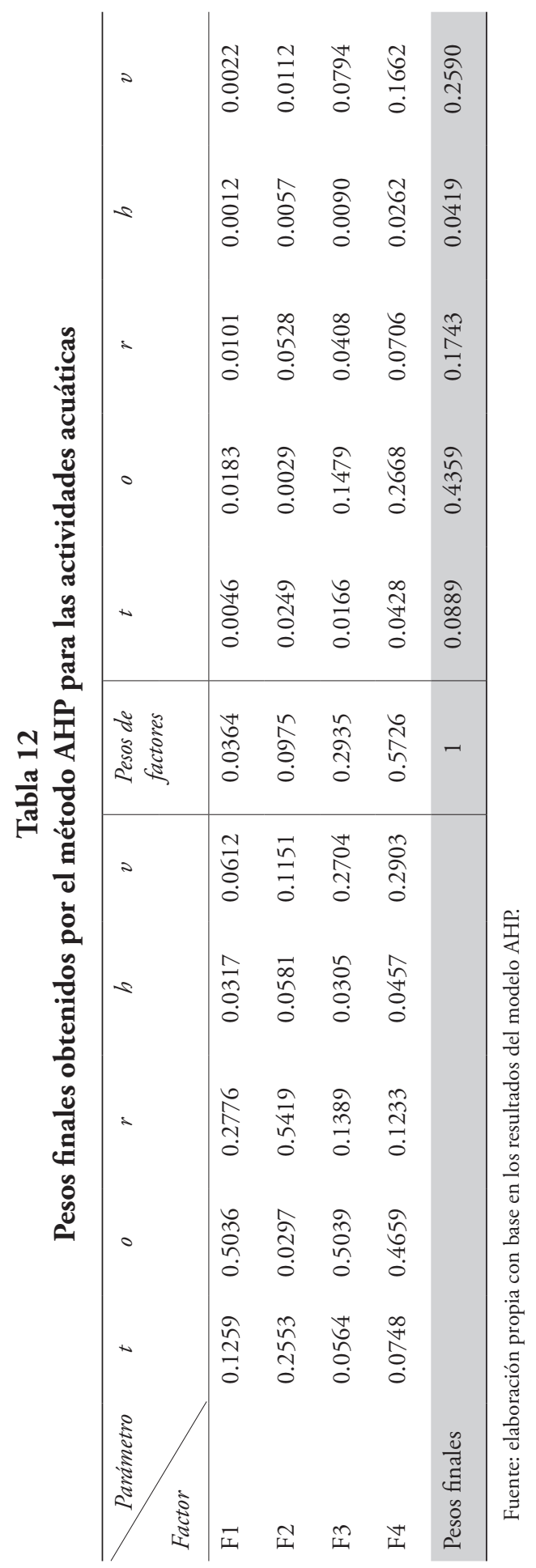


Finalmente, las ecuaciones finales se pueden describir con las siguientes expresiones:

$$
\begin{aligned}
& \operatorname{IAHP}_{f}=\mu(t) 0.1314+\mu(o) 0.3840+\mu(r) 0.3214+\mu(h) 0.0516+\mu(v) 0.1117(13) \\
& \operatorname{IAHP}_{s}=\mu(t) 0.0889+\mu(o) 0.4359+\mu(r) 0.1743+\mu(h) 0.0419+\mu(v) 0.2590
\end{aligned}
$$

En donde los términos $f$ y $s$ corresponden al ambiente familiar y surf respectivamente. La ecuación 13 será utilizada para evaluar playas cuando el determinante sea tener actividades familiares, por lo que playas tranquilas serán mejor evaluadas. La ecuación 14 se empleará cuando el enfoque sea para realizar actividades de surf, y en este caso las playas de mayor oleaje serán mejor evaluadas. La idea principal es que el turista elija su preferencia y de acuerdo con ello, el indicador correspondiente evaluará qué playa es recomendada de acuerdo con su interés. La figura 2 muestra el árbol de jerarquías y los pesos calculados para el cálculo del IAHP ${ }_{f}$.

\section{Figura 2}

Pesos optimizados de los factores y parámetros utilizados para el cálculo del Índice de Evaluación de Costas (IAHP)

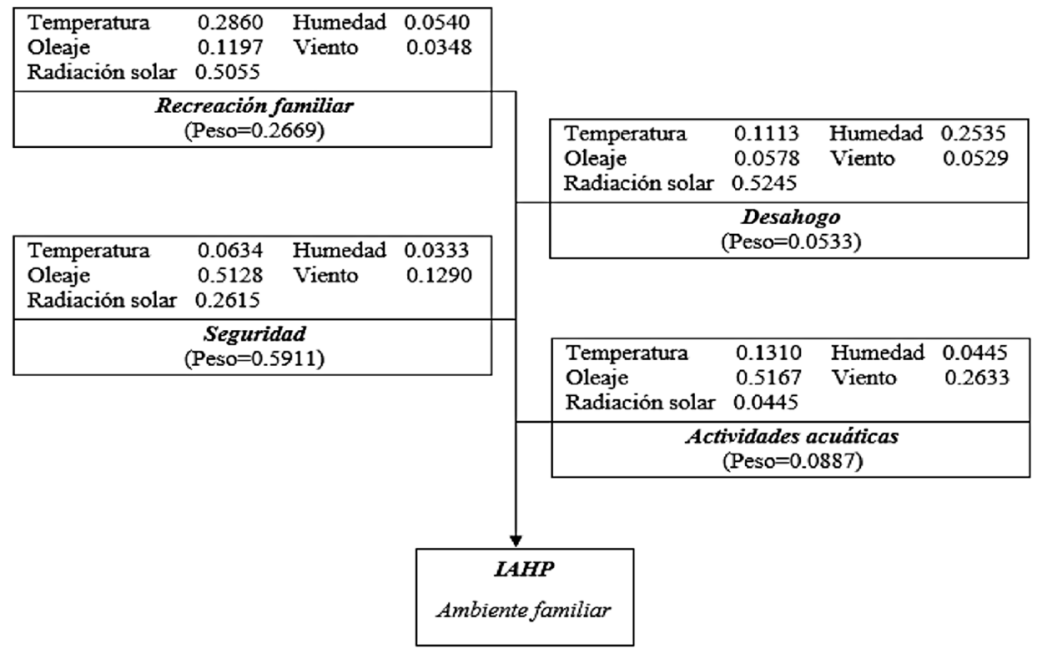

Fuente: elaboración propia. 


\subsection{Adquisición de datos}

Los parámetros de estudio como la temperatura, radiación solar, velocidad del viento y humedad fueron medidos mediante una estación meteorológica Vantage Vue inalámbrica, la cual consta de varios sensores como un anemómetro, capaz de registrar velocidades de viento desde $3 \mathrm{~km} / \mathrm{hr}$ hasta $290 \mathrm{~km} / \mathrm{hr}$, un colector de lluvia, un sensor de temperatura con rango de medición de $-40^{\circ} \mathrm{C}$ a $65^{\circ} \mathrm{C}$, un medidor de humedad relativa y un sensor de radiación. El montaje de la estación se realizó en las instalaciones del Zofemat y del Cicimar, que se encuentran ubicadas en San José del Cabo y La Paz respectivamente. Para la extracción de mediciones de oleaje se emplearon bases de datos proporcionadas por el sitio Windfinder (2019), la cuales tienen una frecuencia de medición de 15 minutos y están disponibles públicamente. Aunque el monitoreo es continuo y automatizado, es necesario analizar un periodo corto para poder observar a detalle el comportamiento de los parámetros y la operación del modelo propuesto. Debido a la sobrecarga de información, 10 días de información fueron evaluadas durante el periodo 2 de junio-12 de junio del 2019 en las playas de La Paz y San José del Cabo, en Baja California Sur. La frecuencia de muestreo se ajustó a 15 minutos por medición durante el periodo, con lo cual se obtuvieron 96 muestras diarias y un total de 1056 muestras por cada parámetro. Esta frecuencia permite observar con precisión el comportamiento de cada parámetro durante el día y a su vez observar momentos ideales durante el día para visita de playa o, por el contrario, qué periodo muestra situaciones no recomendadas.

La gráfica 1 muestra el comportamiento de cada parámetro, así como los límites permitidos (tabla 2). Se pueden apreciar los diferentes comportamientos de los parámetros para cada litoral, en donde la mayoría de éstos sobrepasan los límites óptimos establecidos para cada uno. No obstante, en la sección de oleaje es evidente la gran diferencia entre la ciudad de La Paz y San José del Cabo. Esto se debe principalmente a que las playas de San José tienen la característica de mar abierto, razón por la cual el oleaje es mayor y por consiguiente no recomendables para actividades de natación. 
Gráfica 1

Mediciones de cada parámetro

(del 2 al 12 de junio de 2019), pertenecientes a las costas de La Paz y Costa Azul de San José del Cabo
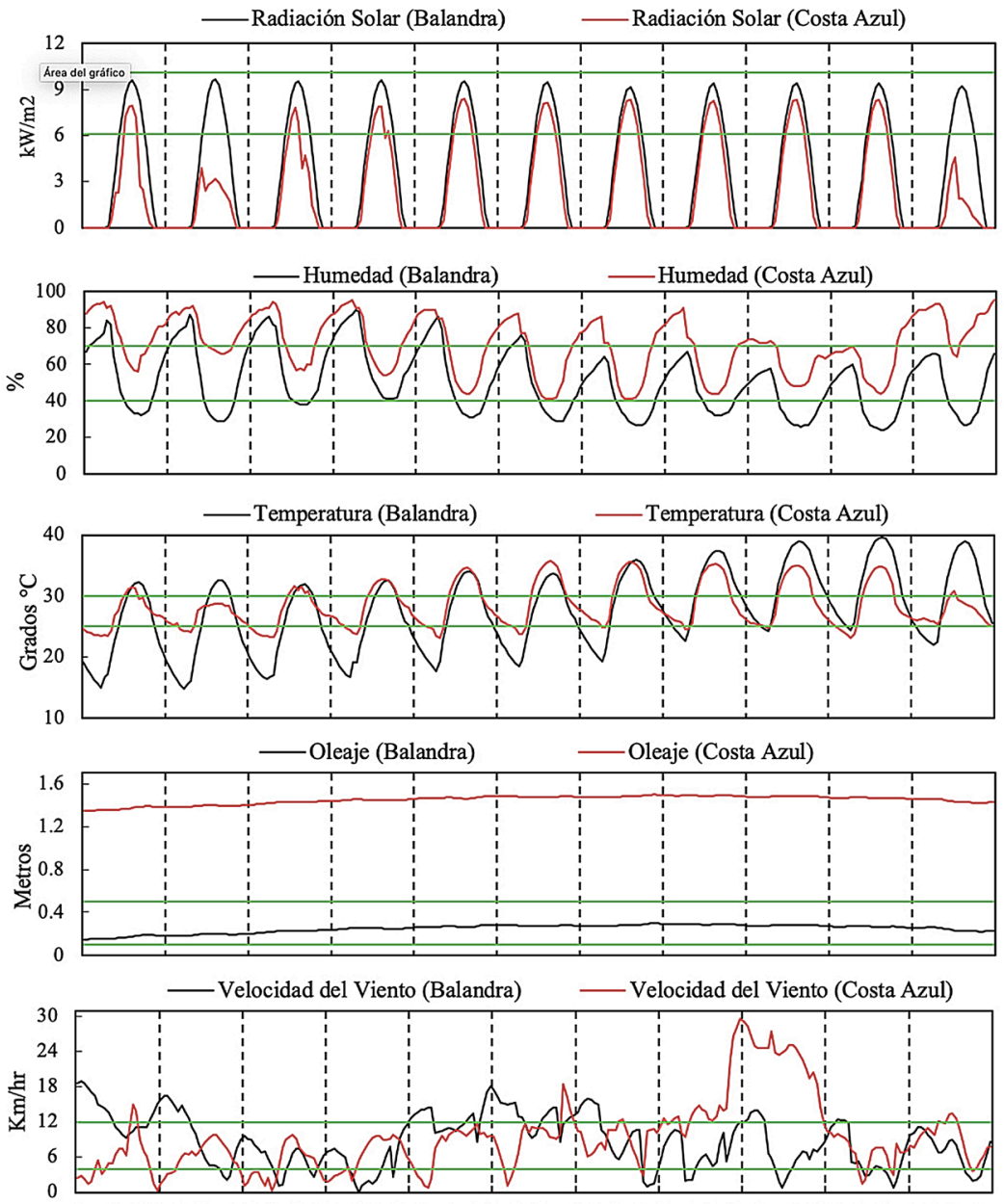

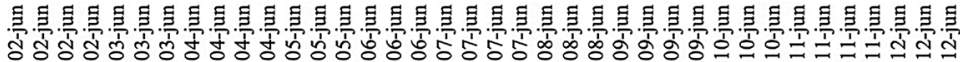

Fuente: elaboración propia con base en datos recolectados durante las prácticas de campo. 


\subsection{Indice de Evaluación de Costas (IAHP)}

El análisis de los sitios de estudio con base en el Índice de Evaluación de Costas (IAHP) se realiza empleando las ecuaciones 13 y 14 y tomando en cuenta los pesos obtenidos en las tablas 13 y 14 . Como entrada, se utilizan las funciones de membresía establecidas en las ecuaciones 1-5. En la literatura, es posible encontrar trabajos similares que permitan obtener evaluaciones de playas y que servirán de base para comparar los resultados obtenidos en el presente trabajo.

El trabajo propuesto por Troyo et al. (2018) utiliza un Índice de Evaluación de Costas Estadístico (IECE), el cual es utilizado para calificar 10 parámetros que interfieren en los servicios relacionados con el avistamiento de la ballena azul y hacer un promedio de éstos para obtener un valor entre 0 y 1 , correspondiente a una mala o buena evaluación respectivamente. Debido a esto, será utilizado para realizar la validación mediante una comparación con nuestro modelo propuesto. En las gráficas 2 y 3 se muestran los resultados de las evaluaciones de las costas de La Paz y San José del Cabo para los casos de recreación familiar y actividades acuáticas.

$\mathrm{Al}$ analizar el caso de las playas ubicadas en La Paz empleando ambos índices, podemos observar que éstos muestran diferentes comportamientos en las dos actividades, lo cual se justifica debido a los intereses y prioridades sugeridos en el modelo AHP. En vista de que la bahía de La Paz presenta bajo oleaje y que el índice para recreación familiar prioriza este parámetro, se puede observar una mejor evaluación en la gráfica 2 (a), con un resultado promedio de 0.58 contra 0.49 obtenido al realizar las mismas evaluaciones con el índice IECE. La diferencia de resultados deriva de la falta de priorización en el modelo, debido a que el IECE calcula el promedio de todos los parámetros, otorgando el mismo nivel de importancia para todo el conjunto en general. Por otra parte, el análisis de la bahía de La Paz que tiene como preferencia la actividad de surf -gráfica 2 (b)-, muestra una clara evaluación negativa con un IAHP promedio de 0.26 y un IECE de 0.29. Esto se puede explicar debido a la falta de oleaje que ahora es un factor negativo para algún turista interesado en surfear. Como se observa en la ecuación 14, este parámetro es importante para la actividad al tener el mayor peso; por consiguiente, restará de la ecuación en mayor medida, razón por la cual obtiene valores más bajos que el IECE.

Al realizar el mismo tipo de evaluación en la ciudad de San José del Cabo, se observa un cambio considerable respecto a la evaluación de las dos actividades. Para la recreación familiar -gráfica 3 (a)-, se obtiene un valor promedio del IAHP de 0.22 contra 0.34 del IECE, esto se debe a que la playa de San José es mar abierto, por lo cual el oleaje presente es 


\section{Gráfica 2}

Resultados del Índice de Evaluación de Costas (IAHP) en la bahía de La Paz para la recreación familiar (a) y práctica de surf (b)
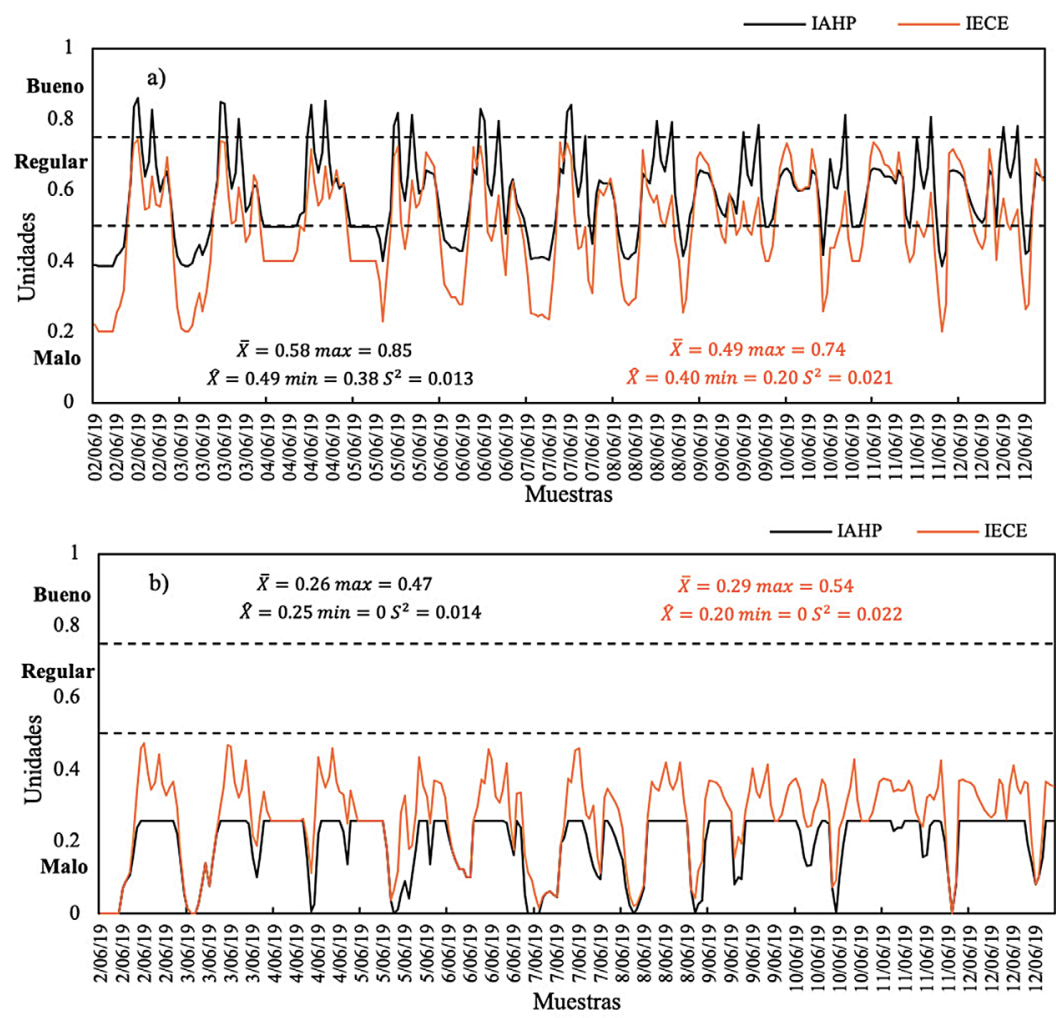

Fuente: elaboración propia con base en los resultados obtenidos.

mayor. Este parámetro es considerado de mayor peso para esta actividad y resulta ser contraproducente en la evaluación, de manera contraria a la evaluación realizada en la playa de La Paz.

En la gráfica 3 (b) se muestran los valores obtenidos para la actividad de surf, donde el IAHP muestra un promedio de 0.72 , una mejor evaluación respecto a lo obtenido en la bahía de La Paz. Lo anterior sucede debido a que la playa de San José presenta mediciones más altas en el oleaje y la velocidad del viento, que son los parámetros de mayor peso para esta actividad. Por otro lado, el promedio obtenido por el IECE es de 0.54 .

Es importante remarcar que la diferencia entre modelos radica en que el índice IECE, al ser un método estadístico, realiza únicamente promedios, lo cual permite compensar el resultado de parámetros peor evaluados 


\section{Gráfica 3}

\section{Resultados del Índice de Evaluación de Costas (IAHP) en Costa Azul para la recreación familiar (a) y práctica de surf (b)}
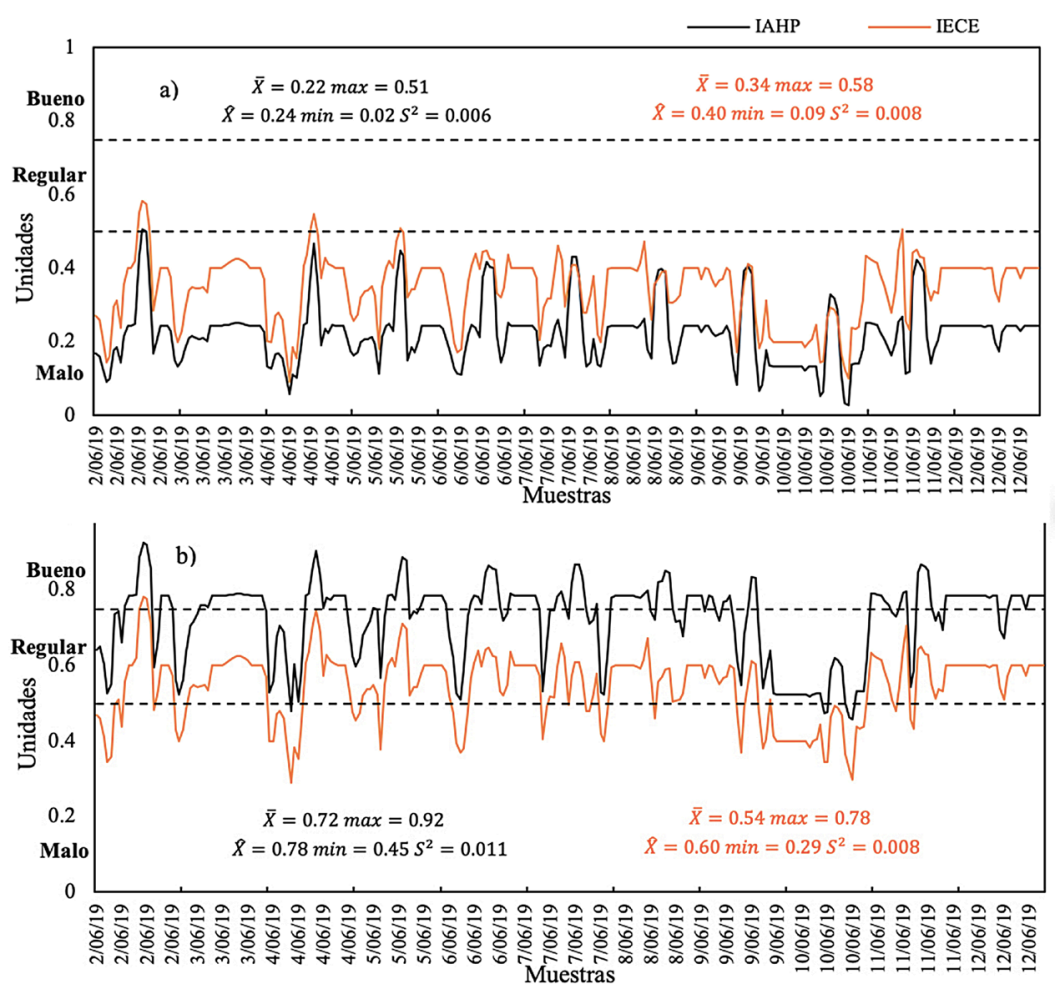

Fuente: elaboración propia con base en los resultados obtenidos.

con aquellos que obtengan una mejor puntuación. En contraste, el índice IAHP permite asignar prioridades a cada factor y parámetros, los pesos se calculan según la actividad de interés, que generan un mayor desplazamiento de los resultados hacia los estados de excelente o malo, generando una evaluación más realista sobre las condiciones que presente cada playa. Con base en lo anterior, podemos deducir que la bahía de La Paz muestra mejores condiciones para actividades familiares, mientras que las playas de San José del Cabo son mejores para realizar actividades de surf. Esto se puede corroborar, toda vez que la zona de San José es mayormente mar abierto y las zonas para nadar están restringidas, cuyo único indicador es una bandera roja en el sitio. De la misma manera, La Paz, al ser bahía, se protege del alto oleaje con una playa que se extiende considerablemente varios metros mar adentro, permitiendo tener uno de los mejores lugares 
para nadar y en donde Bahía Balandra, Pichilingue y el Tesoro son los mejores destinos turísticos de la zona.

De acuerdo con la literatura, aquellos trabajos que analizan características de playa permiten obtener información relevante con respecto en esta temática. Las propuestas de Ergin et al. (2004) y Ergin et al. (2010) permiten evaluar una costa mediante el tipo de vista, indican si algún edificio introduce elementos desagradables o un azul turquesa mejora el paisaje. Asimismo, Ariza et al. (2010) y Espejel et al. (2007) aportan otro tipo de análisis a través de la incorporación de variantes como encuestas check list, mediante las cuales se toman en consideración otros parámetros y factores como la contaminación, la infraestructura, el tipo de arena, entre otros. Sin embargo, estos parámetros son evaluados muy espaciadamente, por lo que su análisis no requiere de un sistema automatizado. Una de las aportaciones de este trabajo es la incorporación de parámetros que pueden monitorearse continuamente y que permiten identificar el estado de la playa en cualquier momento. El análisis de costas fue pensado para analizarse con parámetros cambiantes, ya que el oleaje, viento, humedad, radiación solar y temperatura cambian en todo momento y esto afecta considerablemente el interés por asistir una playa (si hay nubosidad, lluvia, si hace frío, existe alto oleaje o alta radiación UV). Esto permitirá implementar sistemas de monitoreo continuo que permitan a los turistas consultar el estado de una playa dependiendo del clima y características del lugar y poder elegir el lugar de mejor conveniencia para su estancia.

\section{Conclusiones}

En este trabajo presentamos una herramienta de evaluación para el análisis de playas según diferentes factores de interés de cada una de ellas: el Índice de Evaluación de Costas (IAHP). El uso del Proceso Analítico Jerárquico permite tener una flexibilidad al generar criterios de decisión que tengan que ser definidos de manera subjetiva. Para esto se requiere del estudio de actividades que son de gran interés para el turista y de los parámetros que pueden afectar a los mismos. La incorporación de un indicador que permita establecer prioridades acordes con observaciones de expertos en el área ayudará a obtener mayor información de la playa a visitar $y$, asimismo, a implementar acciones que permitan incrementar el nivel de aceptación, toda vez que existe un modelo que permite observar de manera objetiva el comportamiento de un sitio. Este trabajo ha sido realizado con la motivación de no sólo estudiar una bandera de color, la cual se coloca dependiendo de las observaciones del clima, sino de 
establecer un semáforo que permita indicar el adecuado uso de la playa con base en diferentes parámetros que se encuentran involucrados en el lugar. Asimismo, los estudios biológicos permiten saber el estado de contaminación de una playa y sería muy benéfico que este tipo de estudios se realicen constantemente para conocer el estado de salubridad diariamente. Sin embargo, esto requiere de mucho trabajo y recursos tanto humanos como económicos para poder realizarse de esta manera. Por esta razón, los parámetros estudiados pueden medirse automáticamente con el uso de tecnologías que no requieren de mucho esfuerzo para extraer mediciones. El modelo AHP puede ser fácilmente adaptado a un sinfín de variables, siempre y cuando se siga el procedimiento indicado, lo cual permitirá hacer un modelo más completo tomando en cuenta parámetros biológicos y urbanos. De esta manera, este trabajo se propone como una alternativa de apoyo al sector turístico, esperando permita incentivar este sector que en los últimos años ha venido en auge.

\section{Agradecimientos}

Este trabajo ha sido posible gracias al apoyo del Centro Interdisciplinario de Ciencias Marinas (Cicimar), al personal de la Zona Federal Marítimo Terrestre (Zofemat) y a los fondos de investigación SIP-IPN.

\section{Fuentes consultadas}

Abitia-Cárdenas, Leonardo Andrés; Rodríguez-Romero, Jesús; GalvánMagaña, Felipe; de la Cruz-Agüero, José y Chávez-Ramos, Humberto (1994), "Lista sistemática de la ictiofauna de Bahía de La Paz, Baja California Sur, México”, Ciencias Marinas, 20 (2), Mexicali, Universidad Autónoma de Baja California, pp. 159-181.

Adamopoulos, George y Pappis, Costas (1996), "A fuzzy-linguistic approach to a multi-criteria sequencing problem", European Journal of Operational Research, 92 (3), Londres, Elsevier, pp. 628-636.

Ariza, Eduard; Jimenez, Jose; Sarda, Rafael; Villares, Miriam; Pinto, Josep; Fraguell, Rosa; Roca, Elisabet; Marti, Carolina; Valdemoro, Herminia; Ballester, Ramon y Fluvia, Modest (2010), "Proposal for an integral quality index for urban and urbanized beaches", Environmental management, 45 (5), Berlín, Springer, pp. 998-1013. 
Benseny, Graciela (2007), "El turismo en México. Apreciaciones sobre el turismo en espacio litoral", Aportes y transferencias, 11 (2), Mar del Plata, Universidad Nacional de Mar del Plata, pp. 13-34.

Cengel, Yunus y Boles, Michael (2006), Termodinámica (vol. 10), São Paulo, McGraw-Hill.

Chang, Da Yong (1996), "Applications of the extent analysis method on fuzzy AHP”, European Journal of Operational Research, 95 (3), Londres, Elsevier, pp. 649-655.

Cristiano, Samanta da Costa; Rockett, Gabriela Camboim; Portz, Luana Carla; Anfuso, Giorgio; Gruber, Nelson Luiz Sambaqui y Williams, Allan (2016), "Evaluation of Coastal Scenery in Urban Beach: Torres, Rio Grande do Sul, Brazil”, Revista Gestão Costeira Integrada, 16 (1), Lisboa, Associação Portuguesa dos Recursos Hídricos, pp. 71-78.

Dağdeviren, Metin y Yüksel, İhsan (2008), "Developing a fuzzy analytic hierarchy process (AHP) model for behavior-based safety management", Information sciences, 178 (6), Londres, Elsevier, pp. 1717-1733.

DOF (Diario Oficial de la Federación) (2013), "Plan Nacional de Desarrollo 2013-2018”, 20 de mayo de 2013, Ciudad de México, Secretaría de Gobernación. <http://www.dof.gob.mx/nota_detalle.php ?codigo $=5299465 \&$ fecha $=20 / 05 / 2013>, 18$ de octubre de 2019 .

Ergin, Ayşen; Karaesmen, Engin; Micallef, Anton y Williams, Allan (2004), "A new methodology for evaluating coastal scenery: fuzzy logic systems”, Area, 36 (4), Nueva Jersey, Wiley, pp. 367-386.

Ergin, Ayşen; Özölçer, İsmail Hakki y Şahin Fatma (2010), "Evaluating coastal scenery using fuzzy logic: Application at selected sites in Western Black Sea coastal region of Turkey", Ocean Engineering, 37 (7), Glasgow, University of Strathclyde, pp. 583-591.

Espejel, Ileana; Espinoza-Tenorio, Alejandro; Cervantes, Omar; Popoca, Israel; Mejía, Ailin y Delhumeau, Sheila (2007), "Proposal for an integrated risk index for the planning of recreational beaches: Use at seven mexican arid sites", Journal of Coastal Research, (SPEC. ISSUE 50), Florida, Coastal Education \& Research Foundation, pp. 47-51. 
Espinoza, Julio y Rodríguez, Hugo (1987), "Seasonal phenology and reciprocal transplantation of Sargassum sinicola Setchell et Gardner in the southern Gulf of California", Journal of Experimental Marine Biology and Ecology, 110 (2), Ámsterdam, Elsevier, pp. 183-195.

Google (2019), Google Maps, versión 10.23.4., California, Google Inc. <https://www.google.com.mx/maps/>, 12 de octubre de 2019.

Kim, Dae Il; Matsuyama, Yukihiro; Nagasoe, Sou; Yamaguchi, Mimeo; Yoon, Yang Ho; Oshima, Yuji; Imada, Nobuyoshi y Honjo, Tsuneo (2004), "Effects of temperature, salinity and irradiance on the growth of the harmful red tide dinoflagellate Cochlodinium polykrikoides Margalef (Dinophyceae)", Journal of Plankton Research, 26 (1), Oxford, Oxford University Press, pp. 61-66.

Martínez, Genaro y Díaz, Juan José (2011), "Morfometría en la cuenca hidrológica de San José del Cabo, Baja California Sur, México”, Revista Geológica de América Central, núm. 44, San José, Universidad Nacional de Costa Rica, pp. 83-100.

Pérez, Aridio; Céspedes, Carlos y Núñez, Pedro (2008), “Caracterización física-química y biológica de enmiendas orgánicas aplicadas en la producción de cultivos en República Dominicana", Revista de la ciencia del suelo y nutrición vegetal, 8 (3), San José, Universidad de Costa Rica, pp. 10-29.

Pérez, Reyna; Chávez, Plácido y Mancilla, Judith (2016), "Perfil y satisfacción del visitante del destino: Los Cabos, Baja California Sur", Opción, 32 (13), Zulia, Universidad de Zulia, pp. 1041-1068.

Pérez, Reyna (2011), "Globalización en el turismo: Importancia y retos del turismo deportivo, contexto nacional e internacional”, Turydes, 4 (11), Málaga, Universidad de Málaga, pp. 1-12.

Perron, Oskar (1907), "Grundlagen f"ur eine Theore des Jacobischen Kettenbruchalgorithmus", Mathematische Annalen, 64 (1), Heidelberg, Springer, pp. 11-76.

Petrosillo, Irene; Vassallo, Paolo; Valente, Donatella; Mensa, Jean Alberto; Fabiano, Mauro y Zurlini, Giovanni (2010), "Mapping the environmental risk of a tourist harbor in order to foster environmental security: objective vs. subjective assessments", Marine pollution bulletin, 60 (7), Londres, Elsevier, pp. 1051-1058. 
Phillips, Michael Robert y House, Christopher (2009), "An evaluation of priorities for beach tourism: Case studies from South Wales, UK", Tourism Management, 30 (2), Londres, Elsevier, 176-183.

Propin Frejomil, Enrique y Sánchez Crispín, Álvaro (2002), “Estructura regional del turismo en México", Ería: Revista cuatrimestral de geografia, núm. 59, Oviedo, Universidad de Oviedo, pp. 386-394.

Ranieri, Leilanhe Almeida y El-Robrini, Maâmar (2016), "Condição Oceanográfica, Uso e Ocupação da Costa de Salinópolis (Setor CorvinaAtalaia), Nordeste do Pará, Brasil", Revista de Gestão Costeira Integrada, 16 (2), Porto, Universidade do Porto, pp. 133-146.

Rodríguez Brindis, Martín Alberto (2015), "La contribución del Turismo al crecimiento económico de México: un análisis por ramas características del sector", Nova scientia, 7 (13), León, Universidad de La Salle Bajío, pp. 337-351.

Saaty, Thomas (2008), "Decision making with the analytic hierarchy process", International journal of services sciences, 1 (1), Ginebra, Inderscience Publishers, pp. 83-98.

Saaty, Thomas y Wind, Yoram (1980), "Marketing applications of the analytic hierarchy process", Management science, 26 (7), Catonsville, Institute for Operations Research and the Management Sciences (INFORMS), pp. 641-658.

Sectur (Secretaría de Turismo) (2018), "Visión Global del Turismo a México. Análisis de mercados, perspectivas del turismo mundial", Ciudad de México, Sectur.

Semarnat (Secretaría de Medio Ambiente y Recursos Naturales) (2019), "Ficha técnica del análisis del agua", Ciudad de México, Gobierno de México, <https://apps1.semarnat.gob.mx:8443/dgeia/gob-mx/ playas/programas_playas_limpias/ficha_tecnica_analisis_del_ agua.html>, 12 de octubre de 2019.

Toskano Hurtado, Gérard Bruno (2005), "El proceso de análisis jerárquico (AHP) como herramienta para la toma de decisiones en la selección de proveedores", tesis de licenciatura, Universidad Nacional Mayor de San Marcos, Lima. 
Troyo Vega, Benjamín; Arnaud Franco, Gustavo; Galina Tessaro, Patricia; Urbán Ramírez, Jorge; Swartz, Steven y Ortega Rubio, Alfredo (2018), "Evaluación del servicio turístico en el avistamiento de la ballena gris: Baja California Sur, México", Economía, Sociedad y Territorio, 18 (58), Zinacantepec, El Colegio Mexiquense, A.C., pp. 853-880.

UNWTO (World Tourism Organization) (2018), UNWTO Tourism highlights 2018 Edition, Madrid, UNWTO, <https://www.eunwto.org/doi/pdf/10.18111/9789284419876>, 12 de octubre de 2019.

Windfinder (2019), "Previsiones del viento, del oleaje y del tiempo", Kiel, WindFinder.com GmbH \& Co. KG, <https:/www.windfinder. com/forecast/la-paz_baja-california-sur>, 2 de junio de 2019.

Wong, Johnny and Li, Heng (2008), "Application of the analytic hierarchy process (AHP) in multi-criteria analysis of the selection of intelligent building systems", Building and Environment, 43 (1), Londres, Elsevier, pp.108-125.

Wright, Jaime (2008), "Cálculo y mapeo de la radiación solar directa y difusa en Costa Rica”, Uniciencia, 22 (1), San José, Universidad Nacional de Costa Rica, pp. 55-69.

Zofemat (Zona Federal Marítimo Terrestre) (2019), “¿Qué es la Zona Federal Marítimo Terrestre?”, Ciudad de México, <https://www. gob.mx/profepa/acciones-y-programas/que-es-la-zona-federalmaritimo-terrestre-56672>, 17 de octubre de 2019.

Recibido: 27 de noviembre de 2019. Reenviado: 10 de agosto de 2020. Aceptado: 26 de agosto de 2020.

Cesar Augusto Hoil Rosas. Maestro en Ciencias en Ingeniería de Cómputo por el Centro de Investigación en Computación del Instituto Politécnico Nacional (IPN). Actualmente cursa el doctorado en Ciencias de la Computación en el mismo centro, donde lleva a cabo su investigación acerca de la evaluación de litorales turísticos mediante el procesamiento de parámetros climáticos y oceanológicos. Entre sus áreas de interés se 
encuentran la lógica difusa, redes neuronales, procesamiento de señales, electrónica analógica y digital.

José Juan Carbajal Hernández. Maestro y doctor en Ciencias de la Computación por el Centro de Investigación en Computación del Instituto Politécnico Nacional (IPN). Es ingeniero en Comunicaciones y Electrónica por parte de la Escuela Superior en Ingeniería Mecánica y Eléctrica del Instituto Politécnico Nacional (IPN). Es parte del Sistema Nacional de Investigadores nivel 1. Actualmente es profesor e investigador de tiempo completo en el Centro de Investigación en Computación del Instituto Politécnico Nacional, en la Ciudad de México. Sus líneas de investigación incluyen: modelado de sistemas océano-limnológicos, procesamiento de señales, instrumentación analógica y virtual, lógica difusa y redes neuronales artificiales. Entre sus más recientes publicaciones se encuentran, como coautor: "Automatic filter coefficient calculation in lifting scheme wavelet transform for lossless image compression", The Visual Computer, Carouge, Springer Nature, pp. 1-16 (2020); "An Analytical Hierarchy Process to manage water quality in white fish (Chirostoma estor estor) intensive culture", Computers and Electronics in Agriculture, vol. 167, Ámsterdam, Elsevier, (2019), y "Analytical Hierarchy Process Based Model for Safety Assessment of Coastal Touristic Locations", en Jesús Ariel Carrasco-Ochoa, José Francisco Martínez-Trinidad, José Arturo Olvera-López y Joaquín Salas (eds.), Pattern Recognition. 11th Mexican Conference, MCPR 2019 Querétaro, México. Lecture Notes in Computer Science, vol. 11524, Cham Springer, pp. 357-367 (2019).

Luis Pastor Sánchez Fernández. Doctor en Ciencias Técnicas por el Instituto Superior Politécnico "José Antonio Echeverría” de La Habana, Cuba (1998). Profesor-Investigador del Centro de Investigación en Computación del Instituto Politécnico Nacional, México. Es parte del Sistema Nacional de Investigadores nivel 1. Sus intereses de investigación incluyen: Instrumentación virtual, Medición y Procesamiento Inteligente de Variables Físicas, Reconocimiento de Patrones, Medición y Sistemas de Control. Entre sus más recientes publicaciones se encuentran, como coautor: "Fuzzy inference model based on triaxial signals for pronation and supination assessment in Parkinson's disease patients", Artificial Intelligence in Medicine, vol. 105, Ámsterdam, Elsevier (2020); "Soil Moisture Regional Estimation Approach to Determine Irrigation Demands", Computación y Sistemas, 24 (1), Ciudad de México, Instituto Politécnico Nacional, pp. 359-390 (2020), y "Analytical Hierarchy Process Based Model for Safety Assessment of Coastal Touristic Locations", en Jesús Ariel Carrasco-Ochoa, José Francisco Martínez-Trinidad, José Arturo Olvera-López y Joaquín 
Salas (eds.), Pattern Recognition. 11th Mexican Conference, MCPR 2019 Querétaro, México. Lecture Notes in Computer Science, vol. 11524, Cham Springer, pp. 357-367 (2019).

Virginia Berenice Niebla Zatarain. Maestra en Informática y doctora en Ciencias por parte de la Universidad de Occidente. Actualmente se desempeña como Subdirectora de Investigación Científica en el Instituto Tecnológico de los Cabos. Sus líneas de interés son los Sistemas Informáticos aplicados a la industria turística.

Alberto Daniel Dávila Lamas. Maestro en Ciencias en Ingeniería de Cómputo por el Centro de Investigación en Computación. Actualmente es estudiante del doctorado en Ciencias de la Computación, centro donde realiza una investigación sobre la evaluación de la seguridad en litorales empleando modelos de razonamiento difuso. Entre sus áreas de interés se encuentran los modelos de razonamiento difuso, redes neuronales, internet de las cosas y procesamiento digital de señales. 